\title{
Mitochondrial localization of PABPN1 in oculopharyngeal muscular dystrophy
}

\author{
Tsukasa Doki ${ }^{1}$ Satoshi Yamashita $\mathbb{1}^{1} \cdot$ Fan-Yan Wei $^{2} \cdot$ Kentaro Hara $^{1} \cdot$ Takahiro Yamamoto $^{2} \cdot$ Ziwei Zhang $^{1}$. \\ Xiao Zhang ${ }^{1} \cdot$ Nozomu Tawara $^{1} \cdot$ Hirotake Hino ${ }^{3}$. Eiichiro Uyama $\mathbb{C}^{4} \cdot$ Takashi Kurashige $\mathbb{C}^{5,6}$. \\ Hirofumi Maruyama ${ }^{6} \cdot$ Kazuhito Tomizawa $^{2,7} \cdot$ Yukio Ando $^{1,7}$
}

Received: 27 September 2018 / Revised: 9 February 2019 / Accepted: 16 February 2019 / Published online: 20 March 2019

(c) United States \& Canadian Academy of Pathology 2019

\begin{abstract}
Oculopharyngeal muscular dystrophy (OPMD) is a late-onset disorder characterized by ptosis, dysphagia, and weakness of proximal limbs. OPMD is caused by the expansion of polyalanine in poly(A)-binding protein, nuclear 1 (PABPN1). Although mitochondrial abnormality has been proposed as the possible etiology, the molecular pathogenesis is still poorly understood. The aim of the study was to specify the mechanism by which expanded PABPN1 causes mitochondrial dysfunction in OPMD. We evaluated whether transgenic mouse model of OPMD, by expressing expanded PABPN1, indeed causes mitochondrial abnormality associated with muscle degeneration. We also investigated the mechanism by which expanded PABPN1 would cause mitochondrial dysfunction in the mouse and cell models of OPMD. Mitochondrial localization of PABPN1 was observed in the muscle fibers of patients with OPMD. Moreover, abnormal accumulation of PABPN1 on the inner membrane of mitochondria and reduced expression of OXPHOS complexes were detected in the muscle fibers of the transgenic mice expressing expanded human PABPN1 with a 13-alanine stretch. In cells expressing PABPN1 with a 10-alanine or 18-alanine stretch, both types of PABPN1 accumulated in the mitochondria and interacted with TIM23 mitochondrial protein import complex, but PABPN1 with 18-alanine stretch decreased the cell viability and aggresome formation. We proposed that the abnormal accumulation of expanded PABPN1 in mitochondria may be associated with mitochondrial abnormality in OPMD.
\end{abstract}

Supplementary information The online version of this article (https:// doi.org/10.1038/s41374-019-0243-8) contains supplementary material, which is available to authorized users.

Satoshi Yamashita

y-stsh@kumamoto-u.ac.jp

1 Department of Neurology, Graduate School of Medical Sciences, Kumamoto University, Kumamoto, Japan

2 Department of Molecular Physiology, Faculty of Life Sciences, Kumamoto University, Kumamoto, Japan

3 Department of Neurology, Konan Hospital, Kumamoto, Japan

4 Department of Neurology, Kumamoto Takumadai Rehabilitation Hospital, Kumamoto, Japan

5 Department of Neurology, National Hospital Organization Kure Medical Centre, Kure, Japan

6 Department of Clinical Neuroscience and Therapeutics, Hiroshima University Graduate School of Biomedical and Health Sciences, Hiroshima, Japan

7 Center for Metabolic Regulation of Healthy Aging, Faculty of Life Sciences, Kumamoto University, Kumamoto, Japan

\section{Introduction}

Oculopharyngeal muscular dystrophy (OPMD) is a lateonset hereditary and intractable myopathy, which is characterized by progressive ptosis, dysphagia, and weakness of proximal limbs. Taylor first described a French-Canadian family having the disease with vagus-glossopharyngeal paralysis and ptosis in 1915 [1], and Victor et al. later introduced the term OPMD in 1962 [2]. So far, patients with OPMD have been reported in various ethnicities with the highest prevalence in Bukhara Jews (1:700) [3] and FrenchCanadians $(1: 1,000)[4,5]$. In Japan, the precise prevalence of the disease has been undetermined although several case reports were published $[6,7]$.

OPMD is caused by the abnormal expansion of alanineencoding $(\mathrm{GCN}) \mathrm{n}$ trinucleotide repeat in the coding region of the polyadenosine (poly[A]) binding protein nuclear 1 (PABPN1) gene with 11-18 repeats instead of the normal 10 repeats [4]. PABPN1 plays pleiotropic roles, such as the regulation of poly(A) tail length on RNA transcripts $[8,9]$ 
and alternative polyadenylation $[10,11]$, affecting mRNA levels and stability. It is also involved in the long noncoding RNA [12] and small nucleolar RNA processing [13], hyperadenylation and decay of RNA [14] as well as splicing regulation $[15,16]$. However, the precise mechanism by which the small expansion of alanine residues leads to myodegeneration in specific muscle groups remains unclear.

The expanded PABPN1 protein is misfolded and prone to aggregation into intranuclear inclusions, with sequestration of other proteins including RNA-binding proteins, ubiquitin, and heat shock proteins [17-21]. Although the presence of nuclear aggregates is considered to be the main histopathological hallmark of the disease [22], the intranuclear inclusions are detected in only a small fraction of myonuclei in OPMD [23], presumably suggesting that the intranuclear aggregates are not the only pathological mechanism.

Currently, mitochondrial abnormality has been proposed as a possible etiology of OPMD. Previous histological studies have shown mitochondrial changes, such as cytochromec-oxidase (COX)-negative fibers and abnormal ultrastructural findings of mitochondrial with paracrystalline inclusions or enlarged mitochondria with abnormal cristae [24-26]. Certain studies reported that the abnormal mitochondria were a nonspecific epiphenomena in OPMD [27]. In contrast, a recent study showed that the overexpression of PABPN1 with an expanded polyalanine resulted in the upregulation of the proapoptotic protein, p53 and its downstream targets, PUMA and Noxa, associated with the redistribution of p53 to the nucleus and mitochondria and the translocation of Bax to mitochondria followed by the release of cytochrome $\mathrm{c}$ and the cleavage of caspase 3 [28]. A more recent study demonstrated that the down-regulation of mRNAs encoding mitochondrial proteins started at the earliest stages of OPMD, and that the impaired cleavage during nuclear cleavage/polyadenylation followed by active deadenylation of specific mRNAs, involving Smaug and the CCR4-NOT deadenylation complex, led to their destabilization and mitochondrial dysfunction in the Drosophila model of OPMD [29]. However, whether mitochondrial abnormality in OPMD is a primary trigger of muscle degeneration or a secondary event resulting from muscle degeneration in the pathophysiology of OPMD remained undetermined.

So far, several rodent models expressing mutant PABPN1 protein with a $(\mathrm{GCN}) \mathrm{n}$ expansion have already been generated [30-34]. We established the first transgenic OPMD model mouse that expresses mutant hPABPN1 cDNA with a $(\mathrm{GCN})_{13}$ repeat expansion using a chicken $\beta$-actin (CAG) promoter [30, 35]. The transgenic mice line expressing mutant hPABPN1 showed a reduction in the rate of body weight from birth to 4 weeks of age, and a reduced intake of water and food per $24 \mathrm{~h}$, possibly suggesting the presence of dysphagia. The pathological features of skeletal muscles in the mutant line closely resembled those of OPMD patients, such as myopathic changes, rimmed vacuoles, and nuclear inclusions. The histological changes were especially prominent in the levator palpebrae superioris and pharyngeal muscles. The second transgenic mice expressing mutant A13-PABPN1 regulated by the natural promoter showed neither myopathic phenotype nor nuclear inclusion in the muscles [31]. The other transgenic mice expressing mutant A17-PABPN1 using the human skeletal actin promoter displayed progressive muscle weakness and nuclear inclusion but not rimmed vacuoles in the skeletal muscles [32]. Thus, our transgenic OPMD model mouse would be a useful tool for the investigation of molecular mechanism.

Our study aimed to clarify whether our transgenic mouse model of OPMD, by expressing mutated PABPN1, indeed causes mitochondrial abnormality associated with muscle degeneration. We also investigated the mechanism by which mutant PABPN1 would cause mitochondrial dysfunction in the mouse and cell models of OPMD.

\section{Materials and methods}

\section{Patients and muscle biopsies}

The study was approved by the Ethics Committee of the Kumamoto University Hospital. All methods were carried out in accordance with relevant guidelines and regulations. We examined muscle biopsy samples of 12 patients with OPMD and 10 control subjects. In the case of all patients, muscle biopsy and genetic testing were performed for diagnostic purposes after obtaining informed consent. All samples had previously been examined by routine histochemical techniques. Fresh frozen samples were kept at $-80^{\circ} \mathrm{C}$ until used.

\section{Transgenic mouse model of OPMD by expressing mutated PABPN1}

Generation of transgenic mice expressing expanded hPABPN1 in the muscle tissues was described previously [30]. All animal research was approved by the Kumamoto University Committee on Animal Research (No. A28-054, approved at 1/4/2016). All experiments in this study were approved by the ethical committee of the Kumamoto University (Rinri No. 1030, approved at 19/10/2015), and were performed in accordance with the relevant guidelines and regulations required by the Kumamoto University.

\section{Cell culture, transfection, and cell viability}

HEK293F cells were supplied by Thermo Fisher Scientific (Carlsbad, CA, USA). For cell model of OPMD, the cells were transiently transfected with pEGFP-C2 vectors 
expressing wild-type PABPN1 with 10-alanine stretch (EGFP-A10) or mutant PABPN1 with 18 alanine (EGFPA18) (kindly provided by Prof. Guy A. Rouleau, Center for Research in Neuroscience, McGill University, and the McGill University Health Center, Quebec, Canada), or empty vector using Lipofectamine 3000 reagent (Cat. No. L3000001, Thermo Fisher Scientific). Twenty-four, 48, and $72 \mathrm{~h}$ after transfection, the cells were analyzed by a MultiTox-Fluor Multiplex Cytotoxicity Assay kit (Cat. No. G9200, Promega, Madison, WI, USA) according to the manufacturer's instruction.

\section{Immunohistochemical analysis}

The fresh-frozen muscle sections were first studied by using routine histochemical techniques including hematoxylineosin (HE), modified Gomori Trichrome (mGT), COX, and succinate dehydrogenase (SDH) staining. Next, the muscle sections were fixed with $4 \%$ paraformaldehyde and blocked with 5\% normal donkey serum and $0.1 \%$ Triton- $X$ in PBS. The following primary antibodies were used: rabbit antiPABPN1 (1:250 dilution; Cat. No. ab75855, Abcam, Cambridge, MA, USA), and mouse anti-VDAC (1:500 dilution; Cat. No. ab14734, Abcam). Immunolabelled proteins were visualized by Alexa 488- or Alexa 546conjugated secondary antibodies (1:200 dilution; Thermo Fisher Scientific). For immunofluorescence, the sections were counterstained with 4',6-diamidino-2-phenylindole (DAPI; Cat. No. H-1200, Vector Laboratories, Burlingame, CA, USA) and examined by using confocal microscopy (FV1200; Olympus, Tokyo, Japan) or fluorescence microscopy (BZ-9000; Keyence, Osaka, Japan). RGB plot profiles were obtained by using ImageJ $1.50 \mathrm{i}$ software (National Institutes of Health).

\section{Extraction of mitochondrial fraction}

Mitochondria extraction of mouse tissues and cells was based on the previous protocol [36]. In brief, fresh muscle samples from the hindlimb were homogenized in homogenizing buffer (440 mM sucrose, $20 \mathrm{mM}$ MOPS, $1 \mathrm{mM}$ EDTA, and protease inhibitor cocktail, $\mathrm{pH}$ 7.2) and centrifuged at $700 \times g$ for $10 \mathrm{~min}$ at $4{ }^{\circ} \mathrm{C}$. Supernatants were collected and centrifuged at $20,000 \times g$ for $20 \mathrm{~min}$ at $4{ }^{\circ} \mathrm{C}$. The supernatants were discarded and the pellet containing the mitochondria was retained. For mitochondria isolation from cells, the HEK293F cells transfected with vectors were used.

\section{Protein preparation, western blot, and immunoprecipitation analysis}

For the preparation of lysates, skeletal muscle tissues obtained from mice were solubilized with radioimmunoprecipitation assay buffer $(50 \mathrm{mM}$ Tris-HCl, $\mathrm{pH} 8.0 ; 150 \mathrm{mM} \mathrm{NaCl} ; 1 \%$ NP-40; $0.5 \%$ sodium deoxycholate; and $0.1 \%$ sodium dodecyl sulfate) and protease inhibitor cocktail (Cat. No. G6521, Promega). Similar amounts of lysates were quantified with the BCA protein assay kit (Pierce Chemical Co., Rockford, IL, USA) and were subjected to western blotting. For immunoprecipitation of EGFP, we employed the immunoprecipitation kit-GFP-tagged immunomagnetic beads (Cat. No. TB13105, Sino Biological Inc., Shanghai, China). The primary antibodies for the various immunoblots were as follows: rabbit anti-PABPN1 (1:1,000 dilution; Cat. No. ab75855, Abcam), mouse anti-VDAC (1:1,000 dilution; Cat. No. ab14734, Abcam), total oxidative phosphorylation (OXPHOS) WB antibody cocktail (Cat. No. ab110413, Abcam), mouse antiTIM23 (1:2,000 dilution; Cat. No. 611222, BD Biosciences, San Jose, CA, USA), and rabbit anti-TOM20 (1:2,000 dilution, Cat. No. 11802-1-AP, Proteintech, Rosemont, IL, USA). After overnight incubation at $4{ }^{\circ} \mathrm{C}$ with the primary antibody, membranes were probed $\left(1 \mathrm{~h}\right.$ at $\left.25^{\circ} \mathrm{C}\right)$ with the appropriate horseradish peroxidase-conjugated secondary antibody (Agilent, Santa Clara, CA, USA). Bands were visualized by using the ECL Prime Western Blotting Detection System (Cat. No. RPN2232, GE Healthcare, Buckinghamshire, England) with ImageQuant LAS 4000 Mini EPUV (Fujifilm, Tokyo, Japan); and the intensity of bands was quantified by using ImageJ $1.50 \mathrm{i}$ software $(\mathrm{NIH})$.

\section{Proteinase K protection assay}

Isolated mitochondrial fractions $(4 \mathrm{mg} / \mathrm{mL})$ were solubilized in homogenizing buffer $(440 \mathrm{mM}$ sucrose, $20 \mathrm{mM}$ MOPS, $1 \mathrm{mM}$ EDTA, and protease inhibitor cocktail, $\mathrm{pH}$ 7.2). The samples were permeabilized with $0.2 \%$ Triton-X-100 as a control. The samples were then treated with indicated different concentrations of proteinase $\mathrm{K}(\mathrm{PK})$ on ice for $5 \mathrm{~min}$ and the reaction was terminated by the addition of protease inhibitor cocktails. The mitochondrial pellets were washed, and resolved by SDS-polyacrylamide gel electrophoresis (PAGE).

\section{Respiratory complex assay}

To analyze the expression levels of mitochondrial respiratory complex, $125 \mu \mathrm{g}$ of mitochondrial proteins was dissolved in $40 \mu \mathrm{L}$ of buffer ( $1 \mathrm{M}$ aminocaproic acid, $50 \mathrm{mM}$ Bis-Tris, $\mathrm{pH}$ 7.4) and $15 \mu \mathrm{L}$ of $10 \%$ dodecylmaltoside. Samples were centrifuged at $100,000 \times g$ for $15 \mathrm{~min}$ at $4{ }^{\circ} \mathrm{C}$. Supernatants were mixed with $3.75 \mu \mathrm{L}$ of $5 \%$ SERVA Blue G (Cat. No. 35050, SERVA Electrophoresis GmbH, Heidelberg, Germany) dissolved in $1 \mathrm{M}$ aminocaproic acid. Samples were subjected to blue native PAGE (BN-PAGE) using a commercially available $3-12 \%$ Bis-Tris native gels (Thermo Fisher Scientific). 


\section{Electron microscopic analysis}

Muscle specimens were fixed with $10 \%$ paraformaldehyde in $0.2 \mathrm{M}$ sodium cacodylate and $2.5 \%$ glutaraldehyde and post-fixed with $1 \%$ osmium tetroxide in $0.1 \mathrm{M}$ sodium cacodylate for $2 \mathrm{~h}$ at room temperature. After dehydration in a graded series of ethanol and propylene oxide, the samples were embedded in epoxy resin. For light microscopy, $1 \mu \mathrm{m}$ sections of the samples were stained with $1 \%$ toluidine blue. Ultra-thin sections stained with uranyl acetate and lead citrate were observed with a JEM-1400 electron microscope (Nihon-Kohden, Tokyo, Japan).

\section{Analysis of mitochondrial morphology}

Cells seeded to a chamber slide were stained with a mitochondrial potential-independent dye, MitoTracker Red CMH2Xros (1 $\mu$ M, Cat \#. M7513, Thermo Fisher Scientific) $24 \mathrm{~h}$ after transfection, and incubated at $45^{\circ} \mathrm{C}$ for $30 \mathrm{~min}$. After the excess dye was removed, cells were washed with PBS, fixed with 3\% formalin for $15 \mathrm{~min}$, and imaged using confocal microscopy (FV1200; Olympus, Tokyo, Japan). For each sample, the proportion of fragmented mitochondria was counted after observation of 10 random fields of cells.

\section{Extracellular flux analyzer}

Cells were seeded and transfected with plasmids in a XFe24 microplate $\left(1 \times 10^{4} /\right.$ per well), and then cultured with normal medium for $24 \mathrm{~h}$ before mitochondrial function assays, according to the manufacturer's protocol provided with the XFe24 Extracellular Flux Analyzer (Agilent Technologies). Cell media were replaced with conditioned medium (high-glucose DMEM without FBS or sodium bicarbonate) and incubated at $37{ }^{\circ} \mathrm{C}$ without $\mathrm{CO} 2$ for $1 \mathrm{~h}$ before completion of the probe cartridge calibration. The baseline oxygen consumption rate (OCR) were measured with the XFe24 Flux Analyzer. Measurements were performed after injections of three compounds that affect bioenergetics: $1 \mu \mathrm{M}$ oligomycin (Agilent Technologies), $2 \mu \mathrm{M}$ carbonyl cyanide 4(trifluoromethoxy) phenylhydrazone (FCCP, Agilent Technologies) and a mixture of $0.5 \mu \mathrm{M}$ antimycin $\mathrm{A}$ and $0.5 \mu \mathrm{M}$ rotenone (Agilent Technologies).

\section{Statistical analyses}

All values are the mean \pm standard deviation unless stated otherwise. Differences between the means were analyzed by using Student's $t$-test and two-way ANOVA, followed by Tukey's test for multiple comparisons.

\section{Results}

\section{Mitochondrial abnormality and localization of PABPN1 into mitochondria in myofibers with OPMD}

To examine mitochondrial abnormalities in the myopathology of patients with OPMD, a routine series of histological staining was performed. mGT staining revealed several, ragged-red fibers in OPMD patients as shown in previous literatures, although the frequency varies in the patients (Fig. 1a). COX-deficient and SDH-strongly positive fibers were also observed focally in the muscle samples from OPMD (Fig. 1a). To investigate an increase of mitochondria, immunofluorescence study using anti-VDAC (a mitochondrial marker) and anti-PABPN1 antibodies was performed. In the skeletal muscles of OPMD patients, VDAC was stained more intensely in both myofibers, with or without intranuclear inclusions of PABPN1, compared to the control patients (Fig. 1b). Interestingly, PABPN1 was observed not only in the nuclei but also in the cytoplasm of the muscle fibers of OPMD (Fig. 1b). To validate the colocalization between PABPN1 and mitochondria, analysis of RBG plot profiles were performed using image $\mathrm{J}$ software, which demonstrated the peaks of PABPN1 and VDAC coincided at multiple sites in OPMD patients (Fig. 1c). In contrast, the colocalization between PABPN1 and VDAC was not observed in control patients (Fig. 1d). Electron microscopic analysis demonstrated typical intranuclear tubulofilamentous inclusions in muscle specimens from OPMD patients as shown in previous literatures (Fig. 1e, f, arrows). Of note, the tubulofilamentous inclusions were detected even in the perinuclear cytoplasmic region of myofibers with OPMD (Fig. 1g, arrows). We next investigated whether the tubulofilamentous inclusions were present at mitochondria on the electron microscopy observation. Abnormal mitochondria with poorly defined cristae morphology were frequently observed (Fig. 1h, i, arrows) although the tubulofilamentous inclusions were not detected inside mitochondria.

\section{Mitochondrial localization of PABPN1 and reduced expression of OXPHOS complexes in myofibers of the expanded hPABPN1 transgenic mice}

We next examined the mitochondrial abnormality in the transgenic (TG) mice overexpressing expanded hPABPN1 as an OPMD animal model. A routine series of staining of the skeletal muscles showed mitochondrial abnormalities similarly to the skeletal muscles of OPMD patients (Fig. S1). The percentages of COX-deficient and SDH-strongly positive fibers were increased age-dependently in the TG mice, whereas nontransgenic (NTG) at 40 weeks of age mice showed no abnormal fibers (Fig. S1). COX-deficient and 

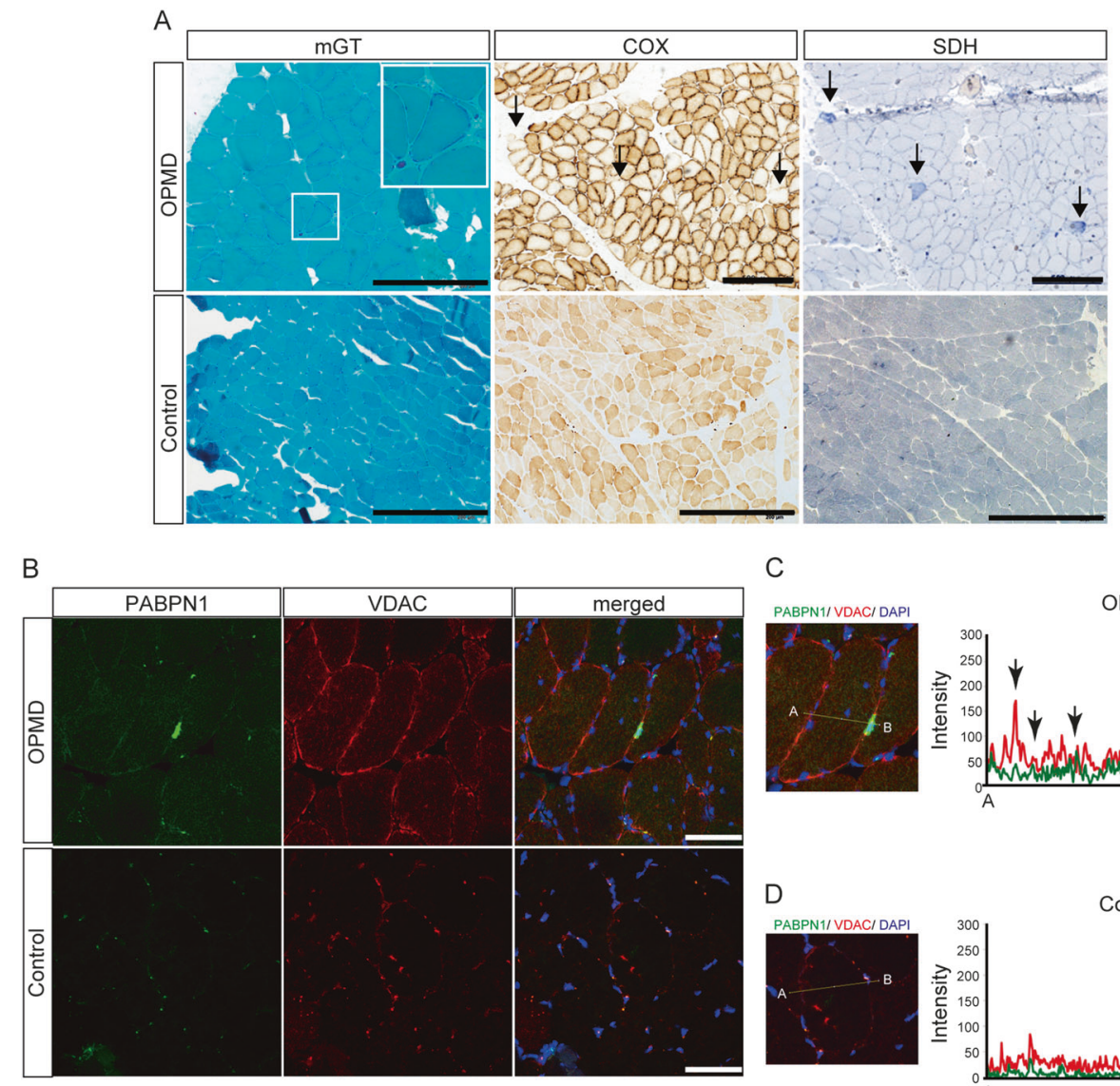

C
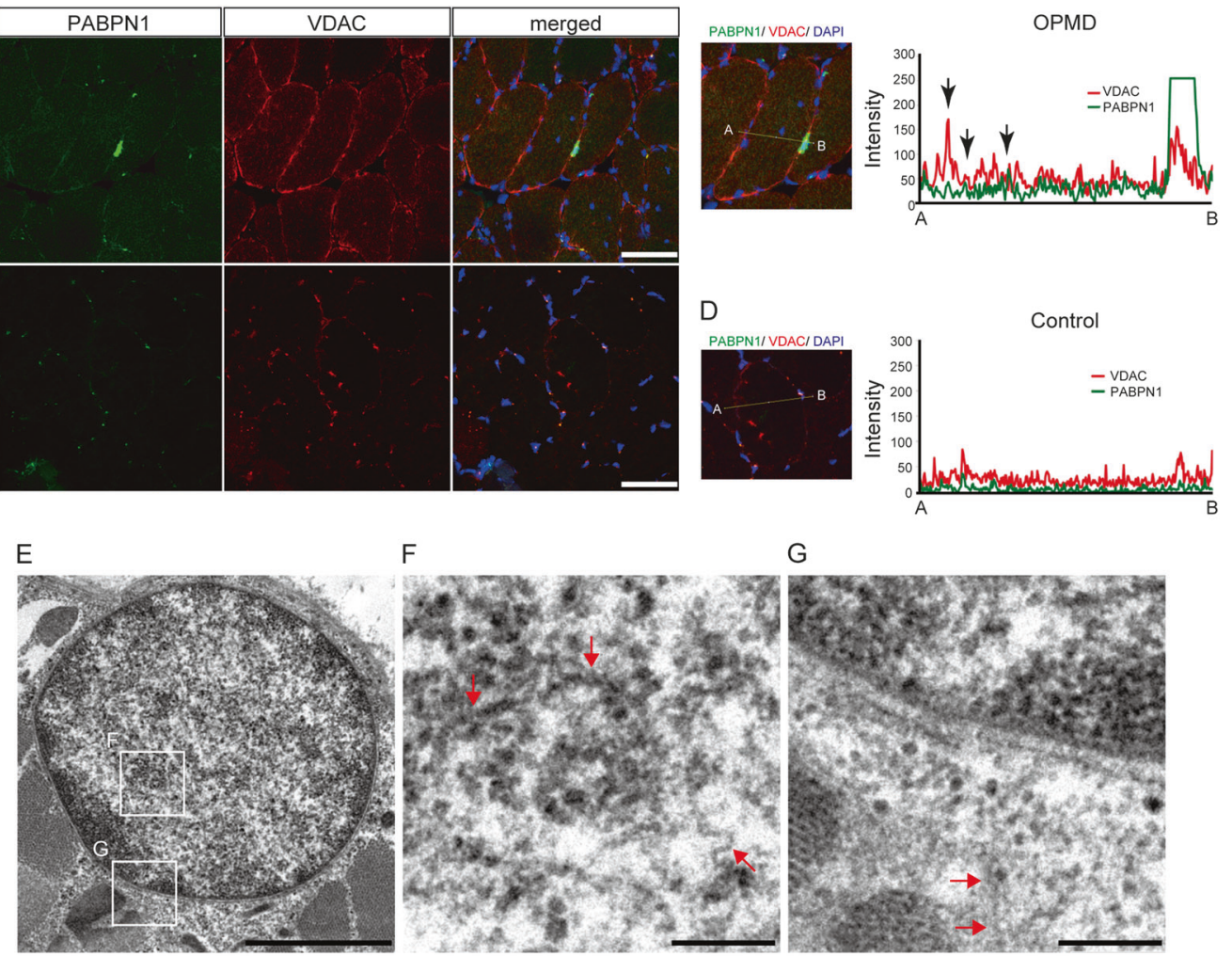

$\mathrm{F}$
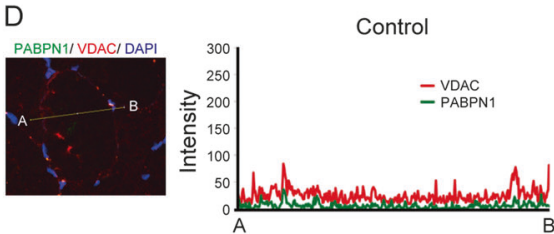

$G$
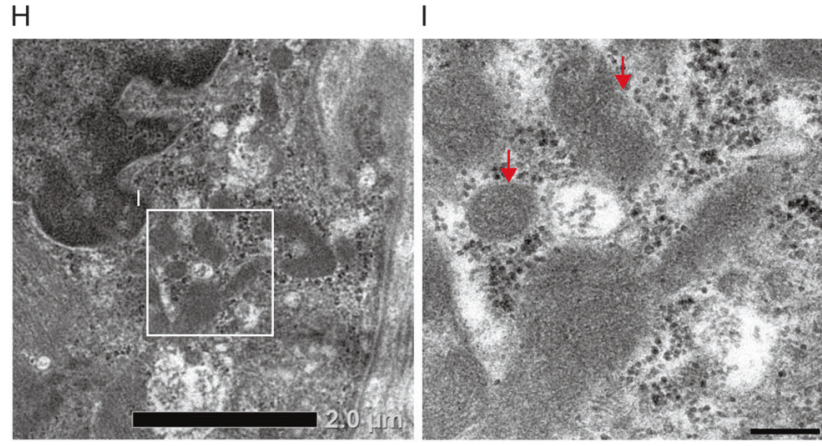

SDH-strongly positive fibers were already observed in 10week old mice, which increased with time, and then the COX signals were almost disappeared in the 40 -week old mice. In immunofluorescence study for VDAC and PABPN1, PABPN1 was observed in both the nuclei and cytoplasm of the myofibers of the TG mice with an age-dependent increase 
Fig. 1 Mitochondrial localization of PABPN1 in myofibers of OPMD patients. a Representative modified Gomori-Trichrome (mGT, left), cytochrome-c-oxidase (COX, middle), and succinate dehydrogenase (SDH, right) staining in the muscle fibers of patients with OPMD (upper) and control patients (lower). Arrows indicate COX-deficient and SDH-strongly positive fibers. Scale bars $=500 \mu \mathrm{m}$. b Immunofluorescence study for PABPN1 (green) and VDAC (red) in the skeletal muscles of patients with OPMD and control patients. Nuclei were counterstained with $4^{\prime}, 6$-diamidino-2-phenylindole (DAPI, blue) in the merged images. Scale bars $=50 \mu \mathrm{m}$. c, d Analysis of RBG plot profiles of the immunofluorescence imaging for PABPN1 (green) and VDAC (red) in OPMD patients (c) and control patients (d). Arrows indicate coincidence of intensity peaks of PABPN1 and VDAC. e-i Electron microscopic analysis in muscle specimens from OPMD patients. Panel $\mathbf{f}$ and $\mathbf{g}$ is higher magnification of panel $\mathbf{e}$. Arrows indicate tubulofilamentous inclusions in the nuclei (f) and perinuclear cytoplasmic region (g). Panel $\mathbf{i}$ is a higher magnification of panel $\mathbf{h}$. Arrows indicate abnormal mitochondria with poorly defined cristae morphology. Scale bars $=2.0 \mu \mathrm{m}(\mathbf{e}, \mathbf{h})$ and $200 \mathrm{~nm}(\mathbf{f}, \mathbf{g}, \mathbf{i})$

in the fluorescence (Fig. 2a). To confirm the colocalization of mitochondria and PABPN1, mitochondrial fractions were isolated from the mouse skeletal muscles, and immunoblot using anti-PABPN1 antibody was performed. PABPN1 was detected in the cytoplasmic and mitochondrial fractions in the 40-week-old TG mice but not in 40-week-old NTG mice (Fig. 2b). To determine the submitochondrial location of mutant PABPN1, a mitochondrial PK protection assay was performed. Mitochondria isolated from the skeletal muscles of the 40-week old TG mice were treated with increasing amounts of $\mathrm{PK}$, and the degradation pattern of mutant PABPN1 was compared with the degradation patterns of mitochondrial proteins representing each compartment of mitochondria (Fig. 2c). TOM20 which localized on the mitochondrial outer membrane was completely digested at PK concentration of $0.13 \mu \mathrm{g} / \mathrm{mL}$, whereas PABPN1 was completely digested at a higher concentration $(0.25 \mu \mathrm{g} / \mathrm{mL}$, Fig. 2c). In contrast, TIM23, which localized on the mitochondrial inner membrane, was completely digested at a much higher concentration ( $1 \mu \mathrm{g} / \mathrm{mL}$, Fig. $2 \mathrm{c})$. The observation suggested that PABPN1 was localized in the mitochondrial intermembrane space. To elucidate the mitochondrial function of the TG mice, the expression levels of OXPHOS complexes were analyzed. The expression levels of all complexes except complex II were decreased in the TG mice, compared to the NTG mice (Fig. 2d). Electron microscopy demonstrated abnormal mitochondria with poorly defined cristae morphology (Fig. 2e-g, arrows) although the tubulofilamentous inclusions were not detected inside mitochondria.

\section{Abnormal accumulation of PABPN1 in mitochondria and interaction with mitochondrial protein import complex, TIM23, in PABPN1-expressing cells}

To investigate the differences between wild-type and expanded PABPN1, we employed cell models expressing the wild-type and expanded PABPN1. HEK293F cells were transfected with EGFP, EGFP with 10-alanine stretch human PABPN1 (EGFP-A10), or EGFP with 18-alanine stretch human PABPN1 (EGFP-A18). To confirm the localization of PABPN1 in mitochondria, the mitochondrial fractions were extracted from cells expressing EGFP, EGFP-A10 or EGFP-A18 and immunoblots were performed using anti-PABPN1 antibody. PABPN1 was detected in both the cytoplasmic and mitochondrial fractions in cells expressing EGFP-A10 and EGFP-A18 (Fig. 3a). PABPN1 gradually increased in the mitochondrial fractions of cells expressing each PABPN1 type with time course (Fig. 3b). To confirm the submitochondrial location of mutant PABPN1, a mitochondrial PK protection assay was performed. TOM20 was completely digested at PK concentration of $0.25 \mu \mathrm{g} / \mathrm{mL}$, whereas PABPN1 was completely digested at a higher concentration $(0.5 \mu \mathrm{g} / \mathrm{mL})$. TIM23 was not completely digested even at a much higher concentration $(2 \mu \mathrm{g} / \mathrm{mL}$, Fig. 3c). These data implied that PABPN1 was localized in the mitochondrial intermembrane space, similar to the PK protection assay using the TG mice. We then investigated the interaction of PABPN1 with mitochondrial complexes by immunoprecipitation with antiEGFP antibody. Anti-EGFP antibody pulled down TIM23, an inner membrane protein of mitochondria, from cell lysates expressing EGFP-A10 and EGFP-A18 (Fig. 3d). In contrast, TOM 20 and VDAC on the outer membrane of mitochondria were not pulled down.

\section{Increased mitochondrial fragmentation in expanded PABPN1- expressing cells}

Mitochondrial morphologies change continuously through the combined actions of fission and fusion events rendering mitochondrial network very dynamic [37]. Fragmented mitochondria often appear at the early phase of cell death associated with permeabilization of the mitochondrial outer membrane [37]. To visualize mitochondrial morphologies, we used a mitochondrial potential-independent dye, MitoTracker Red CM-H2Xros on HEK293F cells transfected with EGFP, EGFP-A10, or EGFP-A18. The proportions of cells with cytosolic PABPN1 were $5.6 \pm 5.2 \%$ and $10.3 \pm$ $4.8 \%$ in cells expressing EGFP-A10 and EGFP-A18, respectively (Fig. 4a, b). Mitochondrial fragmentation was observed more frequently in cells expressing EGFP-A18, compared to cells expressing EGFP-A10 or EGFP alone, or cells with mock treatment (Fig. $4 \mathrm{a}-\mathrm{c}$ ).

We next estimated the effect of accumulation of PABPN1 in mitochondria on the respiration of HEK293F cells transfected with EGFP, EGFP-A10, or EGFP-A18 using the XFe24 Extracellular Flux Analyzer. The baseline OCR in cells with EGFP, EGFP-A10, or EGFP-A18 was lower than that in mock-treated cells, but there was no 
A

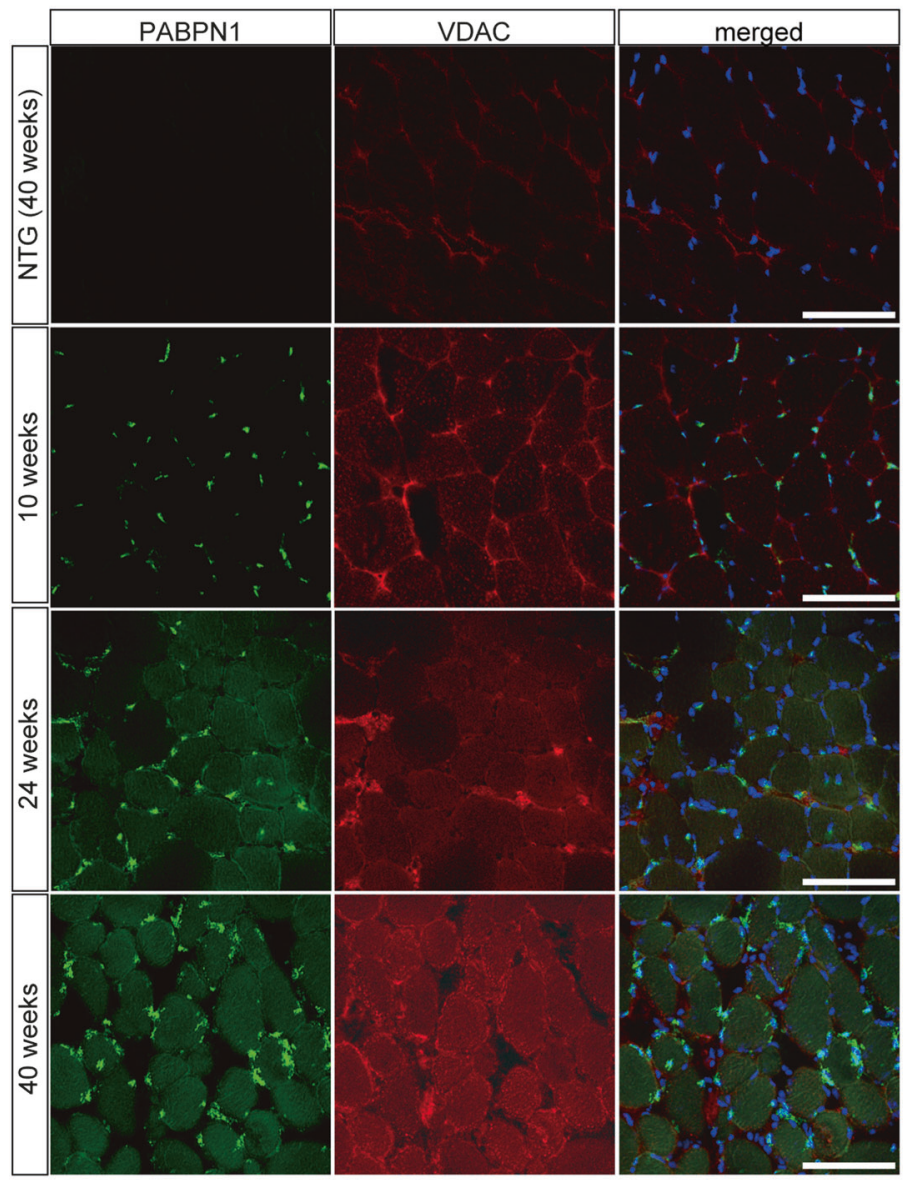

B
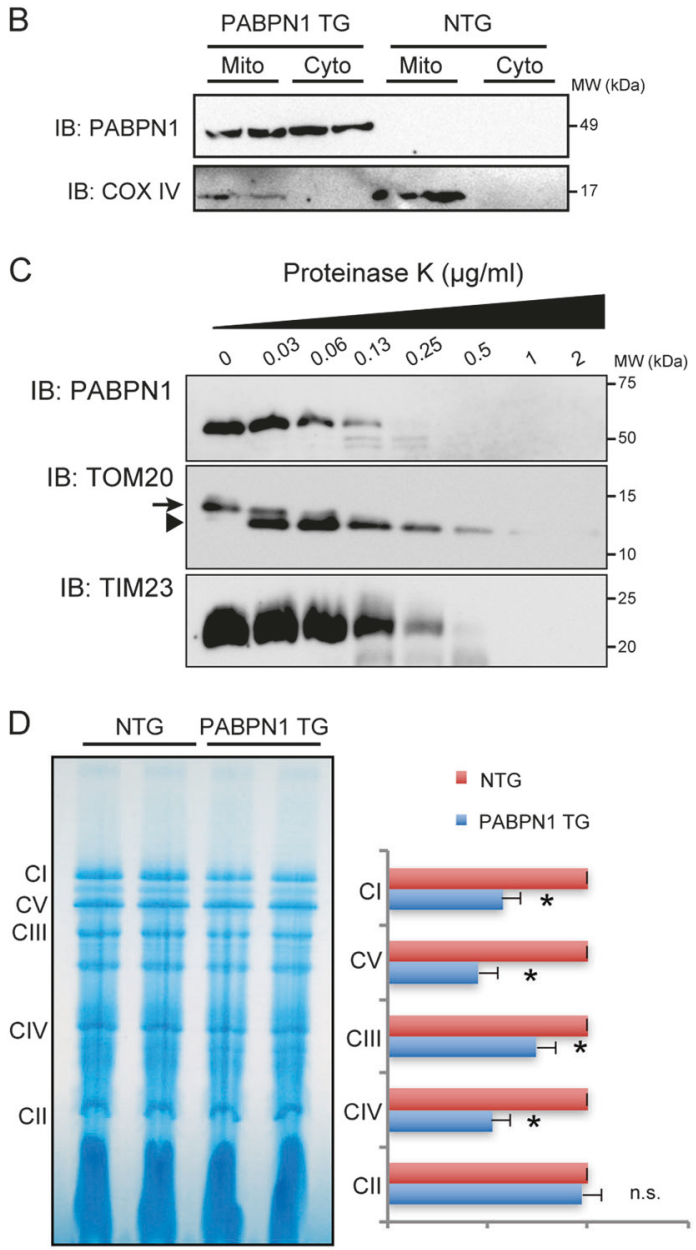

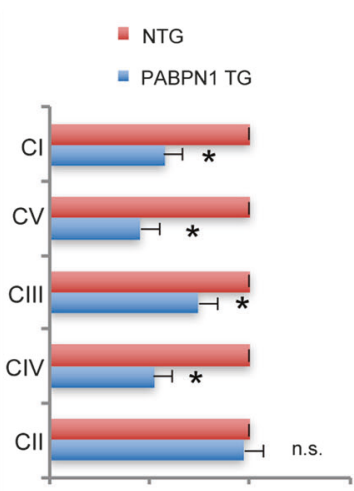

$E$

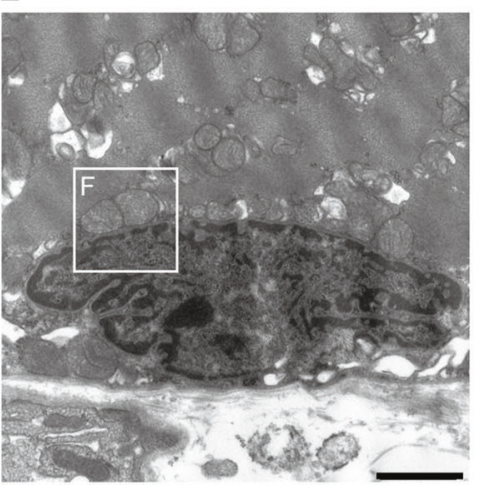

$\mathrm{F}$
G

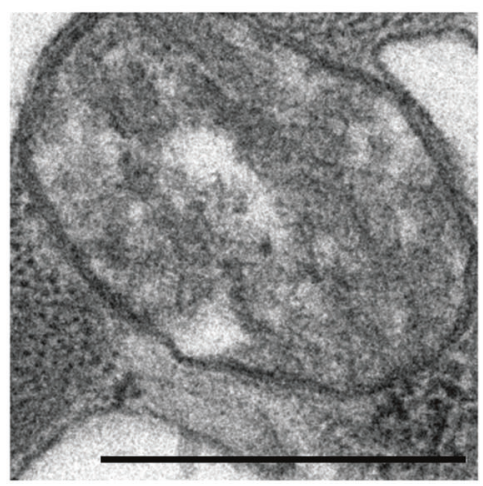

Fig. 2 Mitochondrial dysfunction and localization of PABPN1 into mitochondria in myofibers of the transgenic mice expressing expanded hPABPN1. a Immunofluorescence study for PABPN1 (green) and VDAC (red) in the skeletal muscles of the 10-, 24-, and 40-week-old TG mice, or 40-week-old NTG mice. Nuclei were counterstained with DAPI (blue) in the merged images. Scale bars $=100 \mu \mathrm{m}$. b Immunoblots for PABPN1 and COX IV using the cytosolic (cyto) and mitochondria (mito) fractions of the 40-week-old TG and NTG mice. c Proteinase $\mathrm{K}$ protection assay using the mitochondrial fractions from

difference in the baseline OCR among cells with EGFP, EGFP-A10, and EGFP-A18 (Fig. 4d). We next injected the 40-week-old TG mice with the digestion using indicated concentrations of proteinase K. Arrow indicates the full-length form of TOM20 whereas arrowhead shows the cleaved form. d Blue native PAGE for OXPHOS complexes using the mitochondrial fractions from the 40-week-old TG and NTG mice. ${ }^{*} P<0.05$, n.s., not significant. e-g Electron microscopic analysis in muscle specimens of the 40week-old TG mice. Panel $\mathbf{f}$ is a higher magnification of panel $\mathbf{e}$. Arrows indicate abnormal mitochondria with poorly defined cristae morphology. Scale bars $=1.0 \mu \mathrm{m}(\mathbf{e})$ and $500 \mathrm{~nm}(\mathbf{f}, \mathbf{g})$

oligomycin to inhibit ATP synthase and block ATP production. We then added FCCP to induce maximum OCR by 
A
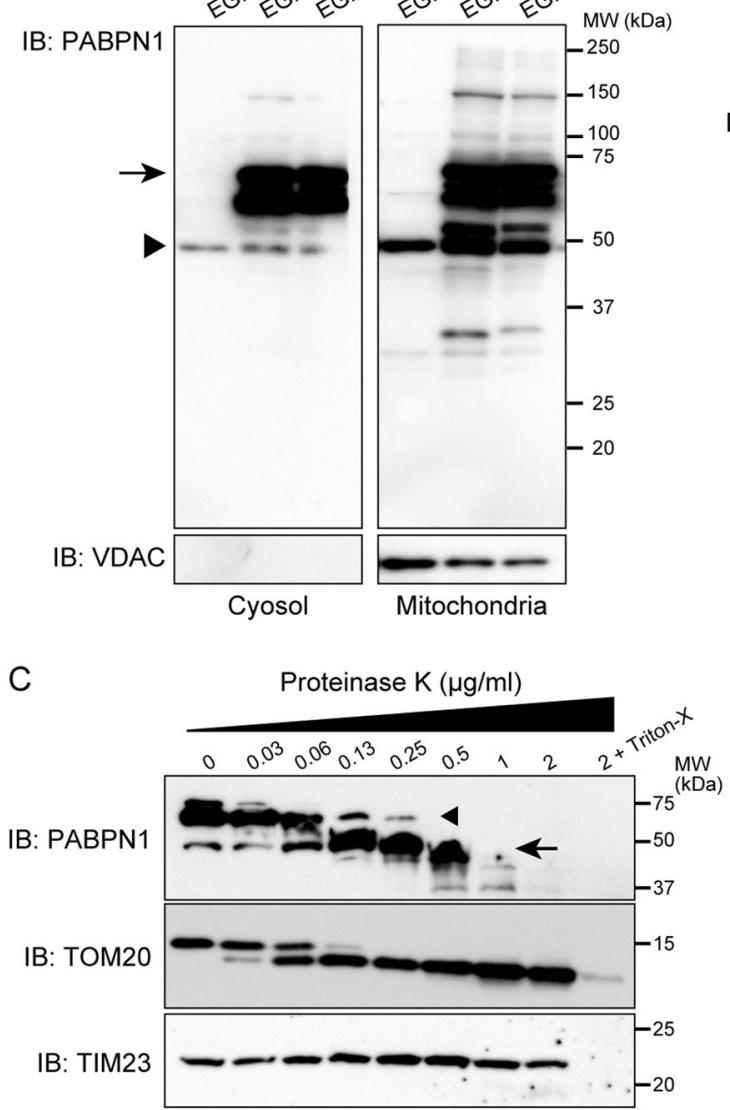

Fig. 3 Abnormal localization of PABPN1 in mitochondria and interaction with TIM23 mitochondrial protein import complex in wild-type and expanded PABPN1-expressing cells. a Immunoblots for PABPN1 and VDAC using the cytosolic and mitochondria fractions of cells transfected with EGFP, EGFP-A10, or EGFP-A18. Arrow indicates EGFP-fused PABPN1 and arrowhead shows endogenous PABPN1. b Immunoblots for PABPN1 and VDAC using the mitochondria fractions of cells expressing EGFP, EGFP-A10, or EGFP-A18 at 24, 48, and $72 \mathrm{~h}$ after transfection. Arrow indicates EGFP-fused PABPN1 and arrowhead shows endogenous PABPN1. Asterisks may indicate cleaved products of PABPN1. c Proteinase K protection assay using

depleting the mitochondrial membrane potential. We finally injected antimycin and rotenone to inhibit the electron transport chain and prevent mitochondria from consuming oxygen. However, we found no significant difference among cells with EGFP, EGFP-A10, and EGFP-A18 during these measurements (Fig. 4d).

\section{Reduced cell viability and aggresome formation in expanded PABPN1- expressing cells}

We next investigated difference in the effect on cell viability between EGFP-A10 and EGFP-A18. Cell viability was significantly reduced in cells expressing EGFP-A18 but not EGFP-A10 (Fig. 5a). Aggresomes are transient microtubule-dependent inclusion bodies that sequester
B

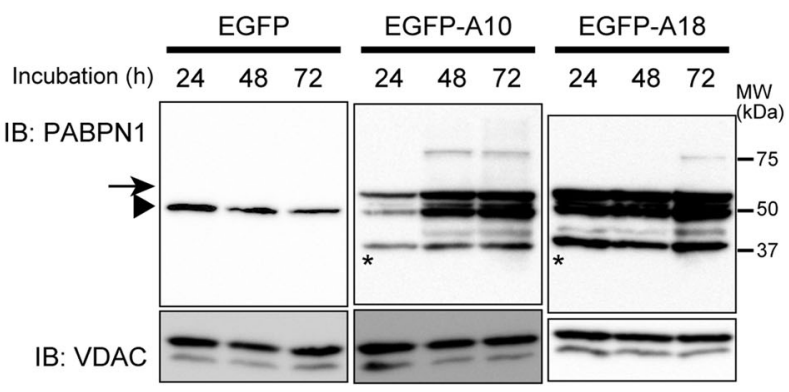

D
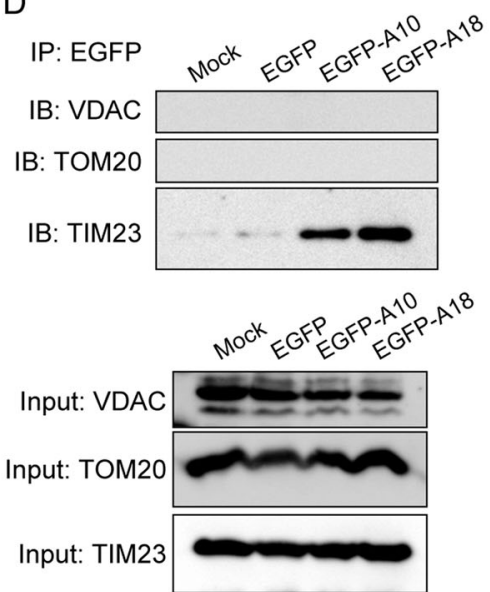

the mitochondrial fractions from the cells expressing EGFP-A18 with the digestion using indicated concentrations of proteinase K. Arrowhead and arrow indicate the full-length and cleaved fragments of PABPN1, respectively. d (Upper panels) Immunoblots for VDAC, TOM20, and TIM23 using output samples immunoprecipitated by anti-EGFP antibodies from the cells expressing EGFP, EGFP-A10, RGFP-A18, or mock. (Lower panels) Immunoblots for VDAC, TOM20, and TIM23 using input samples before immunoprecipitation by anti-EGFP antibodies from cell lysates expressing EGFP, EGFPA10, RGFP-A18, or mock

misfolded proteins and are removed by autophagy. Formation of aggresomes significantly increased in cells expressing EGFP-A18, compared to the cells expressing EGFP-A10 or EGFP alone (Fig. 5b, c), suggesting that EGFP-A18 produced aggregation-prone proteins in the cytoplasm.

\section{Discussion}

We here demonstrated the mitochondrial localization of PABPN1 in the muscle fibers of patients with OPMD. Moreover, the histological changes suggesting mitochondrial abnormality and accumulation of PABPN1 in mitochondria were detected in the muscle fibers of the TG mice 


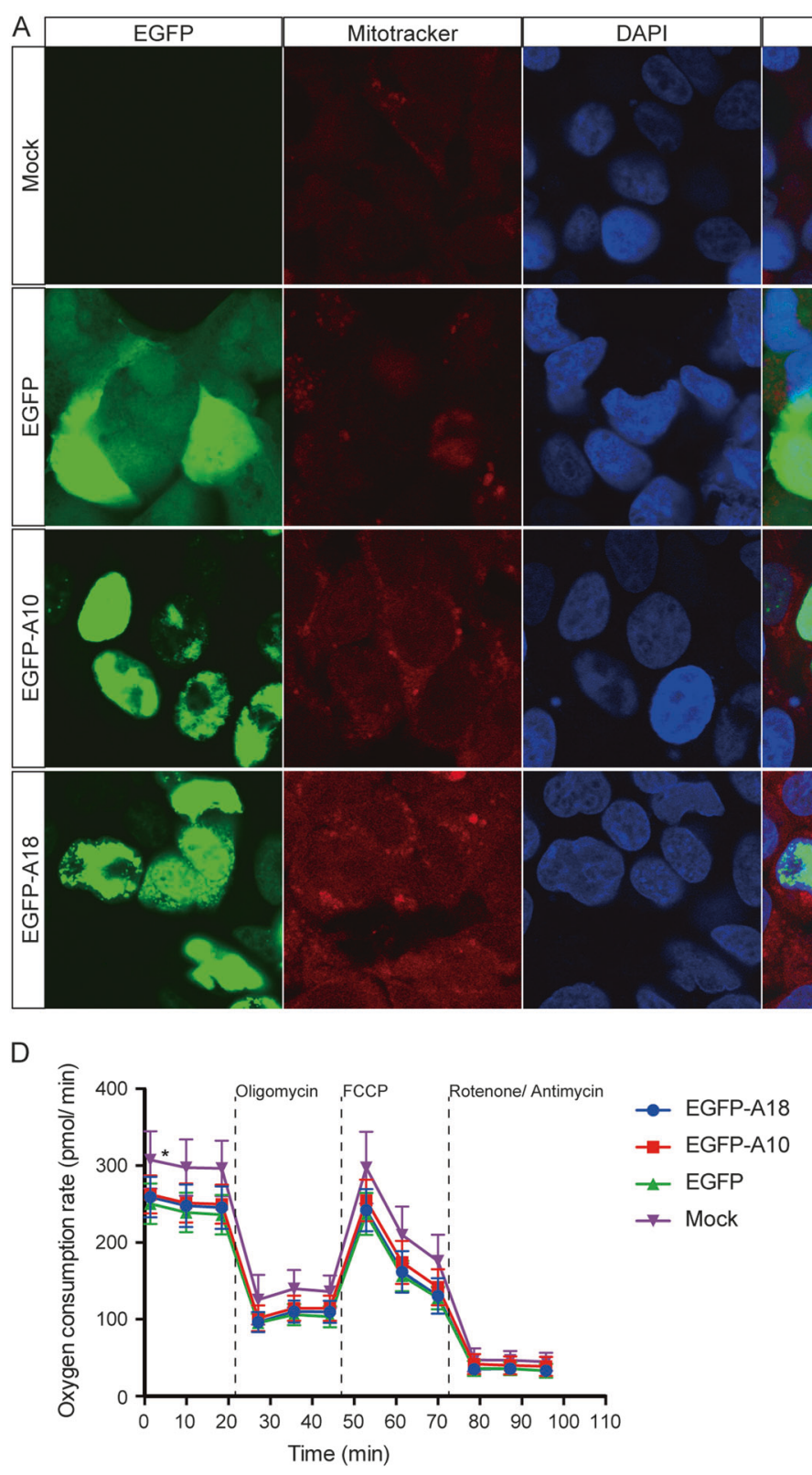

Fig. 4 Increased mitochondrial fragmentation in expanded PABPN1expressing cells. a Immunofluorescence imaging for EGFP (green) and Mitotracker (red) in cells expressing EGFP, EGFP-A10, EGFP-A18, or mock. Nuclei were counterstained with DAPI (blue). Scale bars = $20 \mu \mathrm{m}$. b Percentages of cells with cytosolic PABPN1 in cells expressing EGFP, EGFP-A10, EGFP-A18, or mock. $n=10$ per group. $* P<0.05 ; * * P<0.01 ; * * * * P<0.0001$. c Percentages of cells with

expressing expanded human PABPN1 with 13-alanine stretch. Mislocalization of PABPN1 on the inner membrane of mitochondria and the reduced expression of respiratory chain complex proteins were observed in myofibers of the TG mice. In cells expressing PABPN1 with 10alanine or 18-alanine stretch, both types of PABPN1 were detected in mitochondria and interacted with TIM23 mitochondrial protein import complex, but expanded PABPN1 decreased the cell viability and aggresome formation.
B
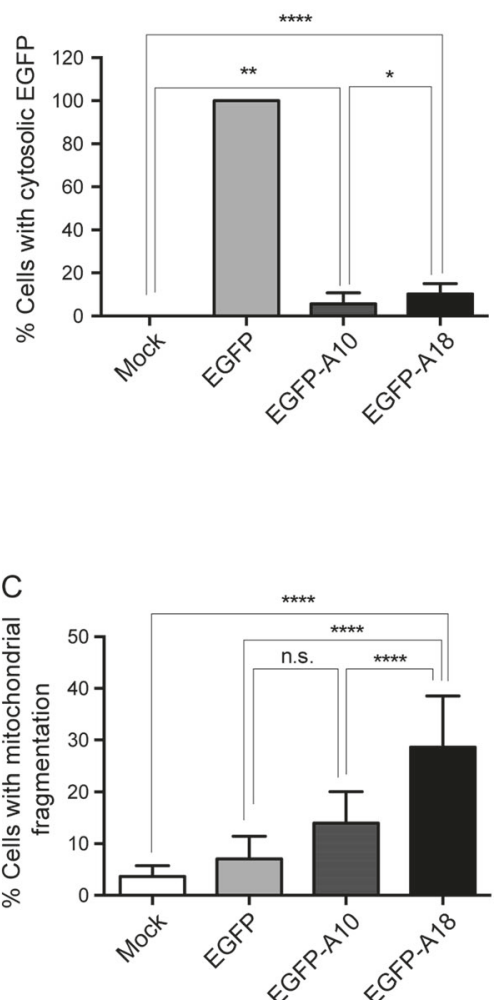

mitochondrial fragmentation in cells expressing EGFP, EGFP-A10, EGFP-A18, or mock. $n=10$ per group. $* * * * P<0.0001$. d Oxygen consumption rate (OCR) measurements in cells expressing EGFP, EGFP-A10, EGFP-A18, or mock. Average experimental OCR measurements at baseline and after addition of oligomycin, FCCP, and rotenone/antimycin are shown. Values are means $\pm \mathrm{SE} ; n=5$ for all conditions. $* P<0.05$

Wild-type PABPN1 can freely shuttle between the nucleus and cytoplasm. Previous studies have shown that the unexpanded wild-type poly(A)-containing proteins localized to the nucleus, but the expanded poly(A)-containing proteins primarily localized to the cytoplasm by interaction with eukaryotic translation elongation factor 1 $\alpha 1$, or by functioning via the expanded poly(A) domain itself as a nuclear export signal [38]. Other studies showed that elongated poly(A) stretches localized exclusively to the 
A

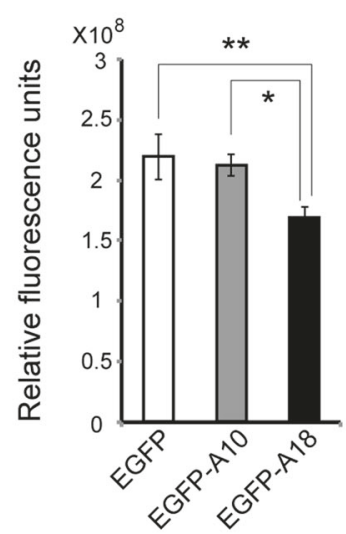

C

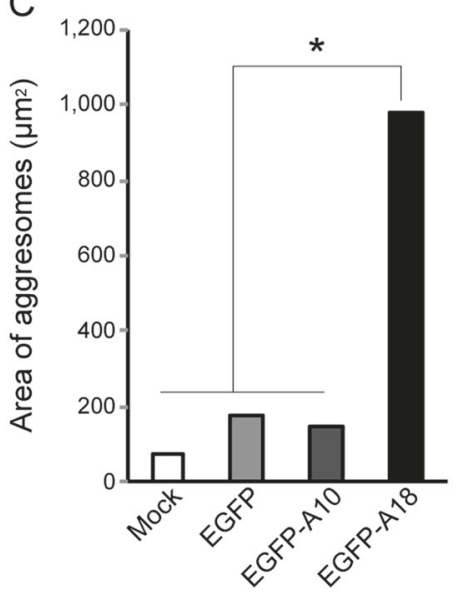

Fig. 5 Decreased cell viability, and aggresome formation in expanded PABPN1-expressing cells. a Cell viability of HEK293F cells transfected with EGFP, EGFP with 10-alanine stretch human PABPN1 (EGFP-A10), or EGFP with 18-alanine stretch human PABPN1 (EGFP-A18). $* P<0.05, * * P<0.01$. b Immunofluorescence imaging for EGFP (green) and aggresome (red) in cells expressing EGFP,

cytoplasm, formed aggregates with mitochondrial proteins, such as succinate dehydrogenase subunit $\mathrm{A}$, and associated directly with mitochondria, reducing the mitochondrial membrane potential [39]. Moreover, it has been shown that oligomerized poly(A) tracts induced the rupture of the mitochondrial membrane, the subsequent release of cytochrome c, and apoptosis [40]. Thus, the expanded poly(A) stretches of PABPN1 itself may induce mitochondrial dysfunction by affecting the expression of mitochondrial proteins and/or mitochondrial membrane potential. However, our observation that both the wild-type (10-alanine) and expanded (18-alanine) PABPN1 localized to the mitochondria and interacted with TIM23 complex, may suggest that the mislocalization of PABPN1 is not associated with expanded poly(A) stretch but attributed to the overexpression of PABPN1 itself.

An interaction between mutant huntingtin with polyglutamine expansion and the TIM23 complex has been

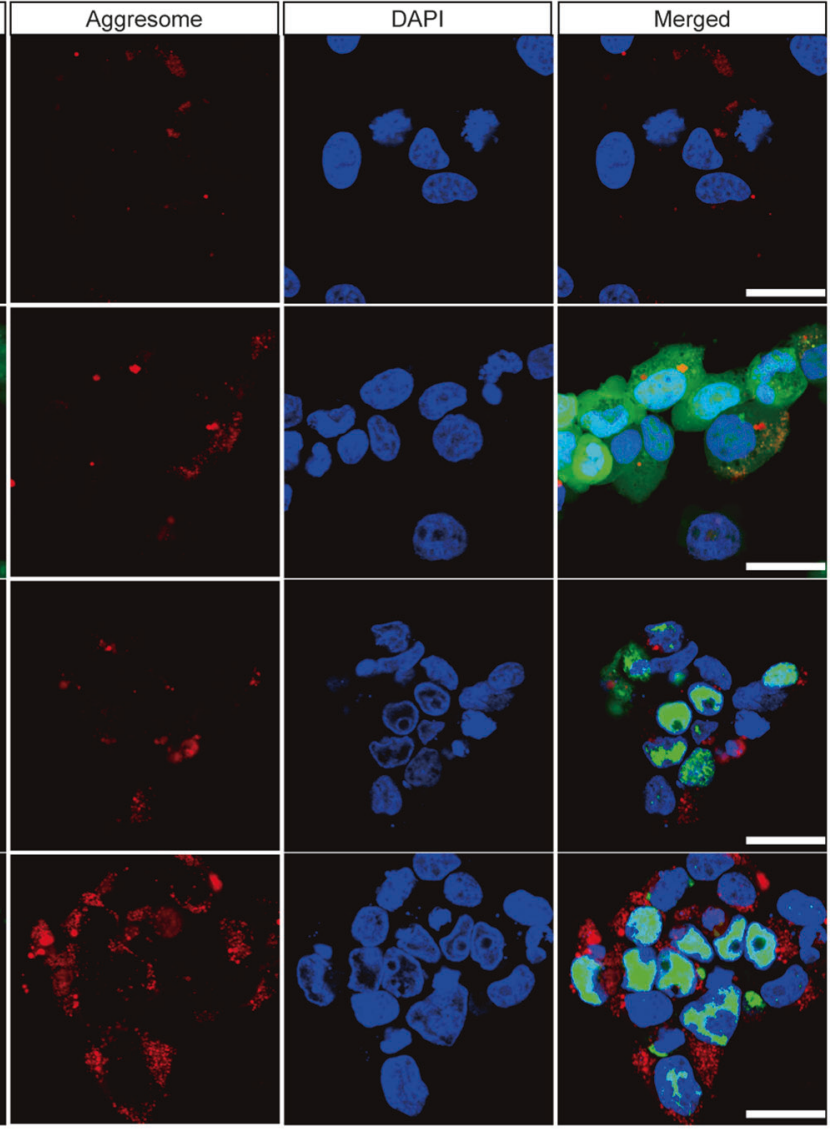

EGFP-A10, EGFP-A18, or mock. Nuclei were counterstained with DAPI (blue). Scale bars $=50 \mu \mathrm{m}$. c Quantification of fluorescence area for aggresomes in cells expressing EGFP, EGFP-A10, EGFP-A18, or mock adjusted, by a uniform threshold using Image $\mathbf{J}$ software. $n=3$ per group. $* P<0.05$

shown in Huntington's disease model, which leads to deficient mitochondrial protein import and subsequent neuronal death [41]. Although we have not showed a direct evidence indicating a deficient mitochondrial protein import in patients and models with OPMD, an interaction between PABPN1 and TIM23 complex may interfere directly with the mitochondrial protein import associated with the pathogenesis of OPMD (Fig. 6).

So far, several mouse models have been generated for understanding of the pathogenesis of OPMD [30-34]. A most recent one is a knock-in mouse model that contained an alanine-expanded Pabpn1 allele under the control of the native promoter and a wild-type Pabpn1 allele [34]. In the model, proteins involved in the mitochondrial metabolism were significantly depleted compared to the wild-type mice, and significantly decreased SDHB (Complex II), COX1 (Complex IV), and ATP5A (Complex V) proteins were detected in the affected muscles from the mice. Thus, the 
A

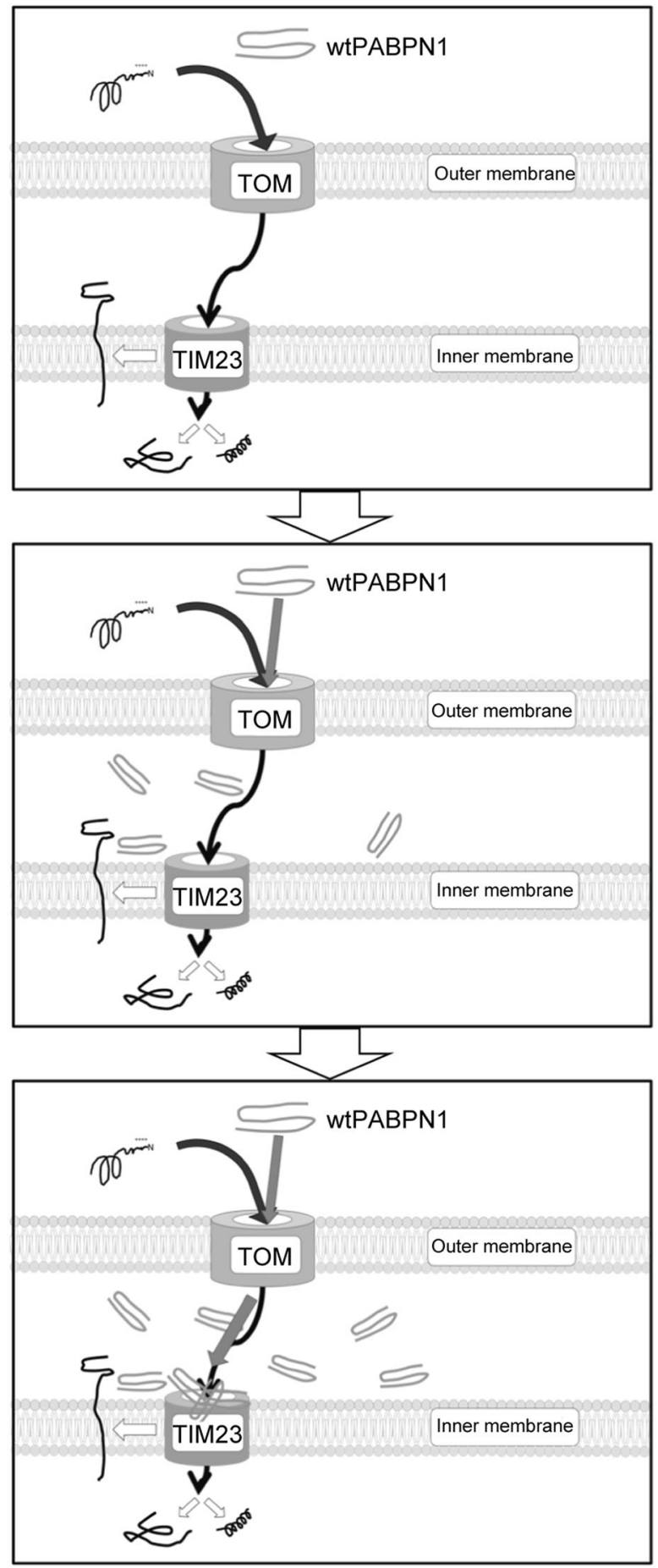

Fig. 6 Schematic images of the pathogenesis of OPMD. a More than $99 \%$ of mitochondrial proteins are encoded by nuclear genes and are synthesized as precursors on cytosolic ribosomes. The cytosolic precursor proteins are recognized by receptors on the mitochondrial surface and are imported by the preprotein translocase of the outer membrane (TOM complex) and/or the inner membrane (TIM23 complex). Wild-type PABPN1 (wtPABPN1) partly localizes in the cytoplasm and enters the intermembrane space of mitochondria.
B

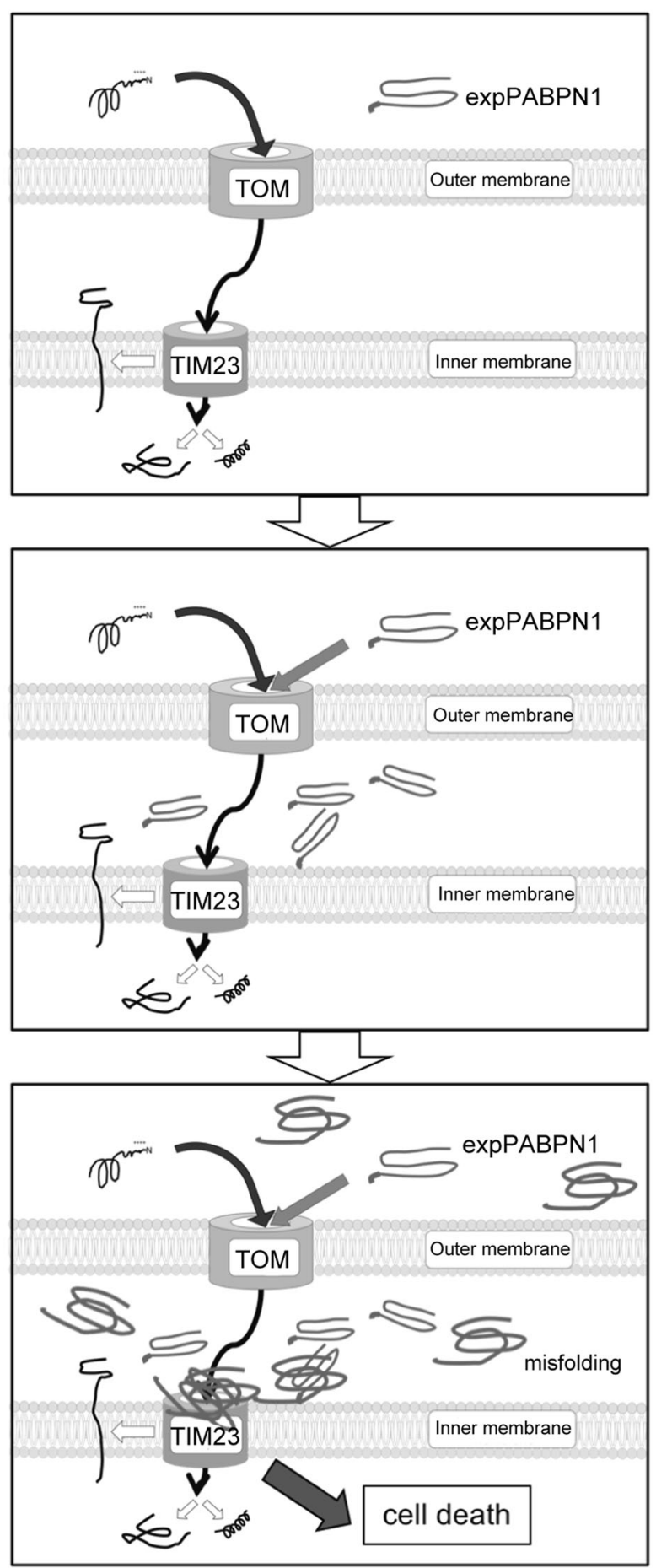

However, the entry of wtPABPN1 into mitochondria does not affect the mitochondria protein transport. b Expanded PABPN1 (expPABPN1) localized not only in the cytoplasm but also in mitochondria. expPABPN1 is more prone to be misfolded than wtPABPN1, and then direct interaction between PABPN1 and TIM23 complex may interfere with the import of mitochondrial inner membrane proteins (black wires) associated with the pathogenesis of OPMD 
dysregulation of electron transport chain and OXPHOS is a common pathomechanism between our TG model and the knock-in model.

OPMD is characterized by myodegeneration in specific muscle groups: the ocular, oropharyngeal, and limb muscles. Chronic progressive external ophthalmoplegia (CPEO) is a mitochondrial myopathy with slowly progressive blepharoptosis, limitation of ocular motility, and dysphagia due to single, large-scale deletions of mitochondrial DNA. Similar phenotypes between OPMD and CPEO may suggest a vulnerability of mitochondrial function in the affected muscles, including the ocular and oropharyngeal muscles.

VDAC1 has been shown to be located not only in mitochondria, but in the sarcoplasmic reticulum and in the sarcolemma in the skeletal muscles [42-44]. The observation of the localization of VDAC revealed the increasing localization in the cytoplasm at the later stage (40-weeks of age) of the TG mice although VDAC staining was mainly in sarcolemma at the earlier stage of the mice, suggesting that the disease stage might affect the number of mitochondria. Mitochondrial abnormality has not been shown in the biopsies of the presymptomatic stage of OPMD in a previous study [45]. Thus, we should give careful consideration to the possibility that mitochondrial dysfunction in OPMD myofibers is not a primary trigger of muscle degeneration but a secondary event resulting from muscle degeneration in the pathophysiology of OPMD.

However, this study has several limitations. Firstly, we have not completely explained the mechanism by which PABPN1 is translocated into the mitochondria, and whether other TOM and TIM complexes are indeed involved in the mechanism. Secondly, the involvement of mitochondrial dysfunction has not been fully evaluated in patients with OPMD; especially the reduced expressions of proteins with mitochondria import signals in proteomic analysis. It is widely known that OPMD has a unique pattern of affecting muscles. We failed to detect a significant difference in OCR in cells with EGFP, EGFP-A10, and EGFP-A18, and the effect of EGFP-A18 expression on cell viability was very limited to approximately $23 \%$ reduction. These results might be attributed to the use of non-muscle cell line. Thus, muscle cell lines would be better to be used although HEK293F cells were employed due to the efficient transfection in the present study.

In conclusion, the abnormal accumulation of PABPN1 with expanded poly(A) in the mitochondria may cause mitochondrial dysfunction in the patients, and animal and cell models for OPMD. Further investigations are required for a better understanding of the roles of mitochondrial dysfunction in the pathogenesis of OPMD.

Acknowledgements This work was supported by a Grant-in-Aid for Scientific Research (24591269) from the Japan Society for the Promotion of Science (JSPS), Japan; and by a Grant-in-Aid for Research on Intractable Diseases from the Ministry of Health, Labour and Welfare of Japan. We thank Dr. Kimi Araki (Division of Reproductive Engineering, Institute of Resource Development and Analysis, Kumamoto University, Kumamoto, Japan) for generation of transgenic mice.

\section{Compliance with ethical standards}

Conflict of interest The authors declare that they have no conflict of interest.

Ethical approval All animal research was approved by the Kumamoto University Committee on Animal Research. All experiments in this study were approved by the ethical committee of the Kumamoto University, and were performed in accordance with the relevant guidelines and regulations required by the Kumamoto University.

Publisher's note: Springer Nature remains neutral with regard to jurisdictional claims in published maps and institutional affiliations.

\section{References}

1. Taylor E. Progressive vagus-glossopharyngeal paralysis with ptosis. A contribution to the group of family diseases. J Nerv Ment Dis. 1915;42:129-39.

2. Victor M, Hayes R, Adams RD. Oculopharyngeal muscular dystrophy. A familial disease of late life characterized by dysphagia and progressive ptosis of the evelids. $\mathrm{N}$ Engl $\mathrm{J}$ Med. 1962;267:1267-72.

3. Blumen SC, Nisipeanu P, Sadeh M, Asherov A, Blumen N, Wirguin Y, et al. Epidemiology and inheritance of oculopharyngeal muscular dystrophy in Israel. Neuromuscul Disord. 1997;7 (Suppl 1):S38-40.

4. Brais B, Bouchard JP, Xie YG, Rochefort DL, Chretien N, Tome FM, et al. Short GCG expansions in the PABP2 gene cause oculopharyngeal muscular dystrophy. Nat Genet. 1998;18:164-7.

5. Hill ME, Creed GA, McMullan TF, Tyers AG, Hilton-Jones D, Robinson DO, et al. Oculopharyngeal muscular dystrophy: phenotypic and genotypic studies in a UK population. Brain. 2001;124:522-6.

6. Uyama E, Nohira O, Chateau D, Tokunaga M, Uchino M, Okabe $\mathrm{T}$, et al. Oculopharyngeal muscular dystrophy in two unrelated Japanese families. Neurology. 1996;46:773-8.

7. Uyama E, Nohira O, Tome FM, Chateau D, Tokunaga M, Ando $\mathrm{M}$, et al. Oculopharyngeal muscular dystrophy in Japan. Neuromuscul Disord. 1997;7(Suppl 1):S41-9.

8. Benoit B, Mitou G, Chartier A, Temme C, Zaessinger S, Wahle E, et al. An essential cytoplasmic function for the nuclear poly(A) binding protein, $\mathrm{PABP} 2$, in poly(A) tail length control and early development in Drosophila. Dev Cell. 2005;9:511-22.

9. Apponi LH, Leung SW, Williams KR, Valentini SR, Corbett AH, Pavlath GK. Loss of nuclear poly(A)-binding protein 1 causes defects in myogenesis and mRNA biogenesis. Hum Mol Genet. 2010;19:1058-65.

10. de Klerk E, Venema A, Anvar SY, Goeman JJ, Hu O, Trollet C, et al. Poly(A) binding protein nuclear 1 levels affect alternative polyadenylation. Nucleic Acids Res. 2012;40:9089-101.

11. Jenal M, Elkon R, Loayza-Puch F, van Haaften G, Kuhn U, Menzies FM, et al. The poly(A)-binding protein nuclear 1 suppresses alternative cleavage and polyadenylation sites. Cell. 2012;149:538-53.

12. Beaulieu YB, Kleinman CL, Landry-Voyer AM, Majewski J, Bachand F. Polyadenylation-dependent control of long noncoding 
RNA expression by the poly(A)-binding protein nuclear 1 . PLoS Genet. 2012;8:e1003078.

13. Lemay JF, D'Amours A, Lemieux C, Lackner DH, St-Sauveur VG, Bahler J, et al. The nuclear poly(A)-binding protein interacts with the exosome to promote synthesis of noncoding small nucleolar RNAs. Mol Cell. 2010;37:34-45.

14. Bresson SM, Conrad NK. The human nuclear poly(a)-binding protein promotes RNA hyperadenylation and decay. PLoS Genet. 2013;9:e1003893.

15. Bergeron D, Pal G, Beaulieu YB, Chabot B, Bachand F. Regulated intron retention and nuclear pre-mRNA decay contribute to PABPN1 autoregulation. Mol Cell Biol. 2015;35:2503-17.

16. Muniz L, Davidson L, West S. Poly(A) polymerase and the nuclear poly(A) binding protein, PABPN1, coordinate the splicing and degradation of a subset of human pre-mRNAs. Mol Cell Biol. 2015;35:2218-30.

17. Fan X, Messaed C, Dion P, Laganiere J, Brais B, Karpati G, et al. HnRNP A1 and A/B interaction with PABPN1 in oculopharyngeal muscular dystrophy. Can J Neurol Sci. 2003;30:244-51.

18. Calado A, Tome FM, Brais B, Rouleau GA, Kuhn U, Wahle E, et al. Nuclear inclusions in oculopharyngeal muscular dystrophy consist of poly(A) binding protein 2 aggregates which sequester poly(A) RNA. Hum Mol Genet. 2000;9:2321-8.

19. Corbeil-Girard LP, Klein AF, Sasseville AM, Lavoie H, Dicaire MJ, Saint-Denis A, et al. PABPN1 overexpression leads to upregulation of genes encoding nuclear proteins that are sequestered in oculopharyngeal muscular dystrophy nuclear inclusions. Neurobiol Dis. 2005;18:551-67.

20. Abu-Baker A, Messaed C, Laganiere J, Gaspar C, Brais B, Rouleau GA. Involvement of the ubiquitin-proteasome pathway and molecular chaperones in oculopharyngeal muscular dystrophy. Hum Mol Genet. 2003;12:2609-23.

21. Tavanez JP, Bengoechea R, Berciano MT, Lafarga M, CarmoFonseca M, Enguita FJ. Hsp70 chaperones and type I PRMTs are sequestered at intranuclear inclusions caused by polyalanine expansions in PABPN1. PLoS One. 2009;4:e6418.

22. Tome FM, Fardeau M. Nuclear inclusions in oculopharyngeal dystrophy. Acta Neuropathol. 1980;49:85-7.

23. Blumen SC, Brais B, Korczyn AD, Medinsky S, Chapman J, Asherov A, et al. Homozygotes for oculopharyngeal muscular dystrophy have a severe form of the disease. Ann Neurol. 1999;46:115-8.

24. Pratt MF, Meyers PK. Oculopharyngeal muscular dystrophy: recent ultrastructural evidence for mitochondrial abnormalities. Laryngoscope. 1986;96:368-73.

25. Pauzner R, Blatt I, Mouallem M, Ben-David E, Farfel Z, Sadeh M. Mitochondrial abnormalities in oculopharyngeal muscular dystrophy. Muscle Nerve. 1991;14:947-52.

26. Gambelli S, Malandrini A, Ginanneschi F, Berti G, Cardaioli E, De Stefano R, et al. Mitochondrial abnormalities in genetically assessed oculopharyngeal muscular dystrophy. Eur Neurol. 2004;51:144-7.

27. Wong KT, Dick D, Anderson JR. Mitochondrial abnormalities in oculopharyngeal muscular dystrophy. Neuromuscul Disord. 1996;6:163-6.

28. Bhattacharjee RB, Zannat T, Bag J. Expression of the polyalanine expansion mutant of nuclear poly(A)-binding protein induces apoptosis via the p53 pathway. Cell Biol Int. 2012;36:697-704.

29. Chartier A, Klein P, Pierson S, Barbezier N, Gidaro T, Casas F, et al. Mitochondrial dysfunction reveals the role of mRNA poly (A) tail regulation in oculopharyngeal muscular dystrophy pathogenesis. PLoS Genet. 2015;11:e1005092.
30. Hino H, Araki K, Uyama E, Takeya M, Araki M, Yoshinobu K, et al. Myopathy phenotype in transgenic mice expressing mutated PABPN1 as a model of oculopharyngeal muscular dystrophy. Hum Mol Genet. 2004;13:181-90.

31. Dion P, Shanmugam V, Gaspar C, Messaed C, Meijer I, Toulouse A, et al. Transgenic expression of an expanded (GCG)13 repeat PABPN1 leads to weakness and coordination defects in mice. Neurobiol Dis. 2005;18:528-36.

32. Davies JE, Wang L, Garcia-Oroz L, Cook LJ, Vacher C, O'Donovan DG, et al. Doxycycline attenuates and delays toxicity of the oculopharyngeal muscular dystrophy mutation in transgenic mice. Nat Med. 2005;11:672-7.

33. Mankodi A, Wheeler TM, Shetty R, Salceies KM, Becher MW, Thornton CA. Progressive myopathy in an inducible mouse model of oculopharyngeal muscular dystrophy. Neurobiol Dis. 2012;45:539-46.

34. Vest KE, Phillips BL, Banerjee A, Apponi LH, Dammer EB, Xu $\mathrm{W}$, et al. Novel mouse models of oculopharyngeal muscular dystrophy (OPMD) reveal early onset mitochondrial defects and suggest loss of PABPN1 may contribute to pathology. Hum Mol Genet. 2017;26:3235-52.

35. Uyama E, Hino H, Araki K, Takeya M, Uchino M, Yamamura K. Animal model of oculopharyngeal muscular dystrophy. Acta Myol. 2005;24:84-8.

36. Wei FY, Zhou B, Suzuki T, Miyata K, Ujihara Y, Horiguchi H, et al. Cdk5rap1-mediated 2-methylthio modification of mitochondrial tRNAs governs protein translation and contributes to myopathy in mice and humans. Cell Metab. 2015;21:428-42.

37. Scholtes C, Bellemin S, Martin E, Carre-Pierrat M, Mollereau B, Gieseler K, et al. DRP-1-mediated apoptosis induces muscle degeneration in dystrophin mutants. Sci Rep. 2018;8:7354.

38. Li L, Ng NK, Koon AC, Chan HY. Expanded polyalanine tracts function as nuclear export signals and promote protein mislocalization via eEF1A1 factor. J Biol Chem. 2017;292:5784-800.

39. Toriumi K, Oma Y, Kino Y, Futai E, Sasagawa N, Ishiura S. Expression of polyalanine stretches induces mitochondrial dysfunction. J Neurosci Res. 2008;86:1529-37.

40. Toriumi K, Oma Y, Mimoto A, Futai E, Sasagawa N, Turk B, et al. Polyalanine tracts directly induce the release of cytochrome c, independently of the mitochondrial permeability transition pore, leading to apoptosis. Genes Cells. 2009;14:751-7.

41. Yano H, Baranov SV, Baranova OV, Kim J, Pan Y, Yablonska S, et al. Inhibition of mitochondrial protein import by mutant huntingtin. Nat Neurosci. 2014;17:822-31.

42. Babel D, Walter G, Gotz H, Thinnes FP, Jurgens L, Konig U, et al. Studies on human porin. VI. Production and characterization of eight monoclonal mouse antibodies against the human VDAC "Porin 31HL" and their application for histotopological studies in human skeletal muscle. Biol Chem Hoppe Seyler. 1991; 372:1027-34.

43. Junankar PR, Dulhunty AF, Curtis SM, Pace SM, Thinnes FP. Porin-type 1 proteins in sarcoplasmic reticulum and plasmalemma of striated muscle fibres. J Muscle Res Cell Motil. 1995;16:595610.

44. Massa R, Marliera LN, Martorana A, Cicconi S, Pierucci D, Giacomini $\mathrm{P}$, et al. Intracellular localization and isoform expression of the voltage-dependent anion channel (VDAC) in normal and dystrophic skeletal muscle. J Muscle Res Cell Motil. 2000;21:433-42.

45. van der Sluijs BM, Raz V, Lammens M, van den Heuvel LP, Voermans NC, van Engelen BG. Intranuclear aggregates precede clinical onset in oculopharyngeal muscular dystrophy. J Neuromuscul Dis. 2016;3:101-9. 